\title{
Modeling and Analysis of Lateral Control System on Electronic Differential for 2-Independent-Wheel Drive Electric Urban Bus
}

\author{
Joga Dharma Setiawan \\ Mechanical Engineering \\ Diponegoro University \\ Semarang, Indonesia \\ joga.setiawan@ft.undip.ac.id \\ Munadi \\ Mechanical Engineering \\ Diponegoro University \\ Semarang, Indonesia \\ muna_096@yahoo.com
}

\author{
Ismoyo Haryanto \\ Mechanical Engineering \\ Diponegoro University \\ Semarang, Indonesia \\ ismoyo2001@yahoo.de \\ Indra Sutanto \\ Mechanical Engineering \\ Diponegoro University \\ Semarang, Indonesia \\ indrasutantoa211@gmail.com
}

\begin{abstract}
To optimize the propulsion performance and to reduce the mass of electric vehicles, an electronic differential subsystem might be preferred since the transmission shaft is removed and the motor is directly connected to wheels. In this research, two motors are used to move 2-Independent-Wheel-Drive of an electric urban bus. In the connection between the two motors, the control system is required to align the performance of both motors to keep the bus stable. This study used the fuzzy logic control in Matlab/Simulink to drive the dynamics of the vehicle modeled in TruckSim. Through calculation, it was found that the maximum lateral speed of the vehicle had the characteristic speed of $150.36 \mathrm{~km} / \mathrm{h}$, with wheel angle conditions and slip angle that occurs are $10 \mathrm{deg}$ and $5 \mathrm{deg}$. However, the speed at which the simulation is used is the maximum speed of the bus with a full charge of $70 \mathrm{~km} / \mathrm{h}$. After the simulation, the difference between the yaw rate is ideal, and the actual yaw rate was compared with the yaw rate range derived from the calculation. In the fuzzy logic control, the difference must be less than the yaw rate range. Simulation results showed that the control system was able to align the 2-independent motors by considering the yaw rate; thus the method can improve the dynamic stability of the bus.
\end{abstract}

Keywords-electric bus, electronic differential, fuzzy logic control, yaw rate control

\section{INTRODUCTION}

Electric buses have the characteristics of zero emission and good economy [1]. Besides, the use of electric buses as public transportation has already widespread in the community, but the safety of the vehicles is one of the problems at this moment. One of the causes of accidents is the incapability of the vehicle when maneuvering. Therefore, the controlled powertrain system of the bus is developed. In this paper, a powertrain model which is used is 2-Independent-Wheel-Drive (IWD), which the bus has two motors on its powertrain.
To control two separate motors working on the bus require an electronic differential. The electronic differential has a role in regulating the voltage applied to the motor according to the given control system.

In the electronic differential, there is a fuzzy logic system that accepts parameters measured through sensors as motor speed, longitudinal speed of the bus, and steering angle. Through the designed control system, the system can determine the required torque as an output of both motors to reach stable conditions.

The using of the electronic differential is more profitable than gear differential because it is lighter and has a fast response. The transmission of the gear on the differential gear makes the time generated in the response become slower. So does the weight, on the differential gear, there is a heavy gearbox that causes its weight exceeds the electronic differential [2].

The main purpose of the electronic differential is to respond to the motor to stabilize the bus during maneuvering. To maintain the stability of the bus, the control in the lateral direction is built. The function of the lateral control system on the bus aims to regulate the torque of the motor in the bus so that the bus could maintain its position of the bus against the load or lateral force applied to the wheel.

Because the existence of two motors that are independent, there is a moment where the torque of both motors do not match with their positions and conditions while the bus maneuvers. To provide an appropriate torque in the maneuvering conditions, the control system on the bus is needed. By controlling the moment that occurs on the bus, the yaw rate of the bus is controlled. Therefore one of the observed parameters from bus condition to make stable in maneuvering is the yaw rate of the bus. 


\section{Direct YAW-Moment CONTROL}

The behavior in maneuvering affects handling performance of the vehicle. In driving, the driver's role and the vehicle are similar to a part of the closed-loop system. However, the driver's concentration in driving will weaken in recent times, and driving mistakes can occur [3]. Direct yaw-moment control function to find out the desired yaw rate which is happening. The purpose of DYC is knowing the desired yaw rate in certain condition so that the desired yaw rate can be controlled to get the desired condition.

Yaw rate that occurs is controlled to be the desired yaw rate by using torque which is produced by motors.

DYC equation located on yaw rate moment control which is shown in Fig 1. After passing TruckSim, the output from the system become the input of the yaw rate moment control. In yaw rate moment control, there are dynamic equations which are used to get the desired yaw rate using the longitudinal velocity and the wheel angle of the bus as the input.

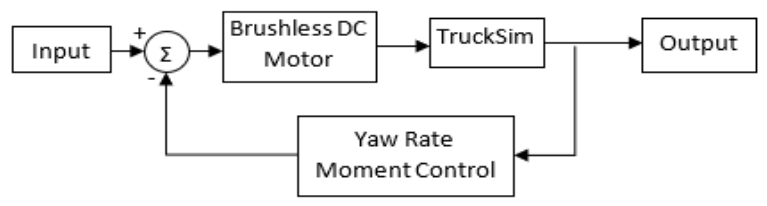

Fig 1 Electronic differential closed-loop system

Vehicle mathematical model used in the simulation is simplified into a bicycle model, both of two wheels at the front and rear of the vehicle become one [4]. This model is showed in Fig 2. In Fig 2, $\alpha_{f}$ and $\alpha_{r}$ respectively are the slip angle of the front and rear wheel. $\delta_{w}$ is steering angle. $L$ as wheelbase.

$l_{f}$ and $l_{r}$ respectively are the distance from the center of the gravity to the front wheel and rear wheel, while $V_{x}$ is the longitudinal speed of the vehicle on the center of mass with the center of rotation $\mathrm{O}$.

$K$ is understeer coefficient, where is according to $K$ value, the trend of the vehicle is known. Subsequently, the maximum velocity can be known, based on the calculation, the maximum speed is characteristic velocity. Equation (4) and (5) are used to find out the tendency or the characteristic of the vehicle, and equation (6) is used to know the yaw rate ratio of the vehicle [5].

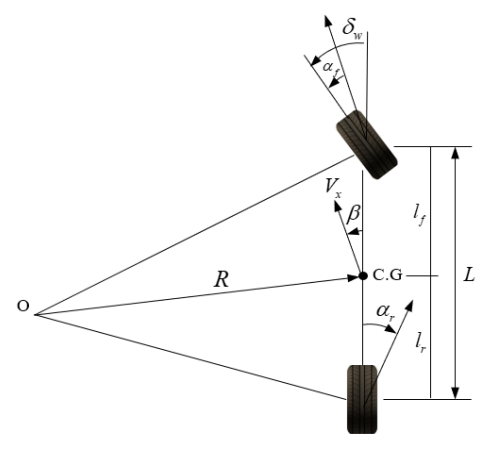

Fig 2 Kinetic and free body diagram of the vehicle

$$
\begin{aligned}
& \dot{\psi}=\frac{V}{R} \\
& \ddot{y}=-\frac{2 C_{\alpha f}+2 C_{\alpha r}}{m V_{x}} \dot{y}+\left(-V_{x}-\frac{2 C_{\alpha f} l_{f}-2 C_{\alpha r} l_{r}}{m V_{x}}\right) \dot{\psi}+\frac{2 C_{\alpha f}}{m} \delta \\
& \ddot{\psi}=-\frac{2 C_{\alpha f} l_{f}+2 C_{\alpha r} l_{r}}{I_{z} V_{x}} \dot{y}-\frac{2 C_{\alpha f} l_{f}^{2}-2 C_{\alpha r} l_{r}^{2}}{I_{z} V_{x}} \dot{\psi}+\frac{2 C_{\alpha f} l_{f}}{I_{z}} \delta \\
& K=\frac{W_{f}}{C_{\alpha f}}-\frac{W_{r}}{C_{\alpha r}} \\
& V_{c h a r}=\sqrt{L g / K} \\
& \frac{r}{\delta}=\frac{V_{x} / L}{1+\frac{K V_{x}^{2}}{L g}} \\
& \delta_{w}=57.3 L / R+\alpha_{f}-\alpha_{r} \\
& r \leq \frac{\mu g}{V_{x}}
\end{aligned}
$$

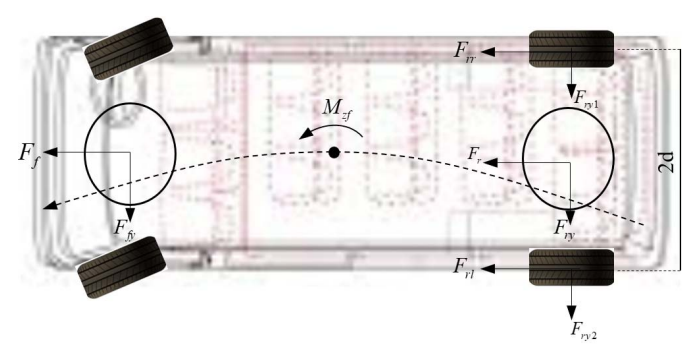

Fig 3 Moment diagram on the bus

$M_{z f}$ is additional yaw moment, which $F_{r r}$ and $F_{r l}$ respectively is the force of rear right and left wheel [6].

$$
M_{z f}=\left(F_{r r}-F_{r l}\right) \cdot d
$$

\section{FUZZY CONTROL STRATEGY}

Fuzzy logic has an advantage which can make decision or output with range $0-1$ by using colloquial language [7]. Parameters controlled in the fuzzy logic system are yaw rate error $(e)$ and yaw rate error rate $(\dot{e})$. Yaw rate error is the error which is happening between the actual yaw rate and the desired yaw rate. To make a decision, fuzzy logic has a few rules for a few ranges of values. Subsequently, input parameters which are accepted are processed by the rule which is made by colloquial language or linguistic variable [8]. 
In the designed fuzzy logic have seven linguistic variables; all of the input variables are NB (Negative Big), NM (Negative Medium), NS (Negative Small), Z( Zero), PS (Positive Small), PM (Positive Medium) and PB (Positive Big).

Desired yaw rate is the yaw rate which is obtained from the mathematical model, and the actual yaw rate is the yaw rate which is happening on the bus at the time.

The output which is produced by fuzzy logic control is the additional moment. By considering both parameters that are yaw rate error and yaw rate error rate, and fuzzy logic rules, therefore, the decision of the additional moment could be executed by using the rules from the TABLE I.

TABLE I. INPUT AND OUTPUT OF FUZZY LOGIC.

Yaw rate error rate

\begin{tabular}{|c|c|c|c|c|c|c|c|}
\hline & NB & $\mathrm{NM}$ & NS & Z & PS & $\mathrm{PM}$ & PB \\
\hline NB & PB & PB & $\mathrm{PM}$ & $\mathrm{PM}$ & PS & PS & Z \\
\hline NM & PB & $\mathrm{PM}$ & $\mathrm{PM}$ & PS & PS & Z & NS \\
\hline NS & PB & $\mathrm{PM}$ & PS & PS & Z & NS & $\mathrm{NB}$ \\
\hline $\mathrm{Z}$ & $\mathrm{PB}$ & $\mathrm{PM}$ & PS & $\mathrm{Z}$ & NS & $\mathrm{NM}$ & $\mathrm{NB}$ \\
\hline PS & $\mathrm{PM}$ & PS & $\mathrm{Z}$ & NS & $\mathrm{NM}$ & $\mathrm{NM}$ & $\mathrm{NB}$ \\
\hline $\mathrm{PM}$ & $\mathrm{PM}$ & PS & $\mathrm{Z}$ & NM & $\mathrm{NM}$ & NB & NB \\
\hline PB & PS & $\mathrm{Z}$ & NM & NM & NB & NB & NB \\
\hline
\end{tabular}

For example, yaw rate error which is happening is NS (Negative Small) or small yet negative, and yaw rate error rate which is happening is NB (Negative Big) or big yet negative, then the decision needed for eliminating the yaw rate error is $\mathrm{PB}$ (Positive Big).

The fuzzy inputs for fuzzy logic shown in Fig 4 and Fig 5 are the yaw rate error and the yaw rate error rate by using the seven linguistic variables in range values between -1 to 1 .

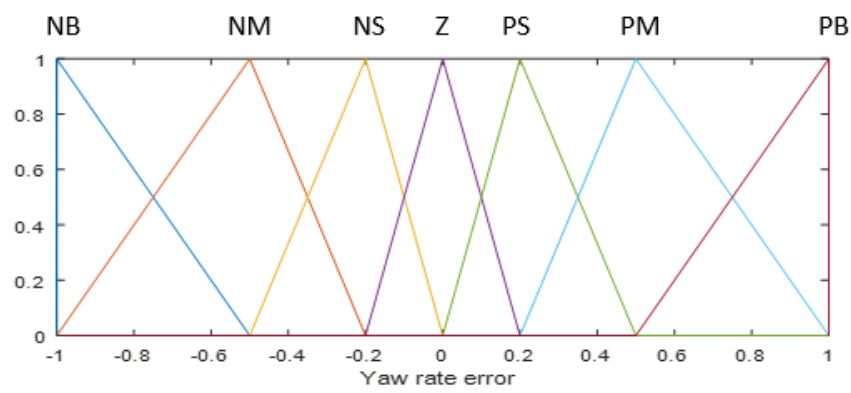

Fig 4 Input variable yaw rate error

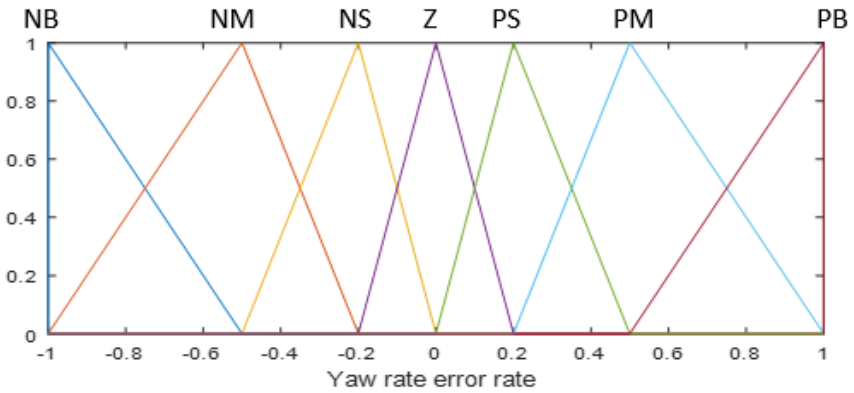

Fig 5 Input variable yaw rate error rate

Through both inputs according to TABLE I, the output is an additional moment. Fig 6 is the fuzzy output for the fuzzy logic system.

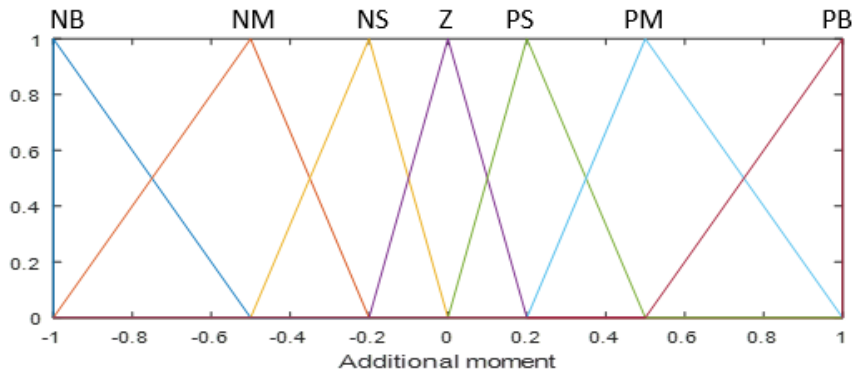

Fig 6 Output variable additional moment

\section{TORQUE CONTROL BASED ON YAW RATE}

Controlling motor speed is the control commonly used in motor control. In this paper, the control which is given to the motor is a torque control method. The torque is controlled to increase the stability of the bus.

Motors used in the powertrain system of the electric urban bus is a permanent brushless direct current motor (BLDC) with $75 \mathrm{~kW}$ power. The direct current motor is a motor which needs one direction current to operate it. BLDC motor is one of the synchronous motors, which means the magnetic fields produced by the stator and the rotor are in the same frequency.

The control of motor has the same response as the motor model using resistance and inductance. From the fuzzy logic system, the additional moment which is needed for the stability condition of maneuvering bus is found. To give an additional moment, the control of motor has a role to adjust the amount of the torque which is given from the motor to the wheel to stabilize the bus through the force given to the wheel both in longitudinal and normal direction.

$$
\begin{aligned}
& e_{a} \quad=v_{b}+i_{a} R_{a}+L_{a} \frac{d i_{a}}{d t} \\
& I_{a}=\frac{1}{R_{a}+L_{a} s}\left(E_{a}-V_{b}\right)
\end{aligned}
$$


$K_{b}=\frac{v_{b}}{\omega_{n}}=0.00684 \frac{\tau}{I_{a}}$

$T_{e}=K_{t} I_{a}=C_{T} \varphi_{N} I_{a}=9.55 C_{e} \varphi_{N} I_{a}$

$Z=\sqrt{R^{2}+X_{L}}$

$X_{L}=\omega L_{a}$

$E_{a}$ is armature voltage $(\mathrm{V}) ; I_{a}$ is armature current $(\mathrm{A}) ; R_{a}$ and $L_{a}$ respectively are resistance and inductance. $V_{b}$ is backelectromotive force voltage $(\mathrm{V}) . K_{b}$ is back-electromotive constant. $K_{t}$ is torque constant. $C_{e}$ is motor potential coefficient $\left(\mathrm{V} / \mathrm{r} \cdot \mathrm{min}^{-1}\right)$.

\section{SIMULATION RESULT AND ANALYSIS}

By using the mathematical model of the dynamics of the bus, the yaw rate ratio for normal and understeer condition can be obtained. The difference of both results is the scale which is used to regulate the range in fuzzy logic control of the yaw rate so that the yaw rate could be confined to the condition got before. In the simulation, the input velocity of the bus is constant at $70 \mathrm{~km} / \mathrm{h}$.

$\left.\frac{r}{\delta}\right|_{n}=5.33 /\left.s \quad \frac{r}{\delta}\right|_{u}=4.38 / s$

$\left|r_{n}-r_{u}\right|=12.635 \mathrm{deg} / \mathrm{s}$

By using equation (4) and (5), the maximum velocity of the bus can be known that is $150.36 \mathrm{~km} / \mathrm{h}$. Whereas, by using equation (7), the values of steering angle for normal and understeer condition in its characteristic speed can be obtained.

In this paper, the simulation is done by using two motors independently. TABLE II gives the main parameters which are used in the simulation. The values in TABLE II are based on the field measurement, observation, and estimation on the shuttle bus shown in Fig 7. The bus operates between Diponegoro University (UNDIP) and Semarang State University (UNNES) in Semarang, Indonesia.

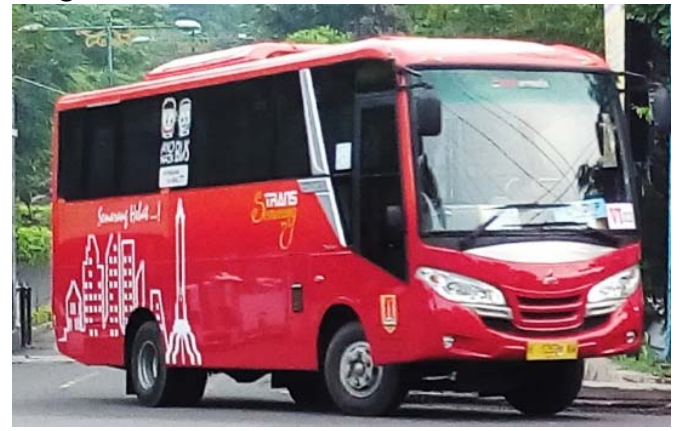

Fig 7 Campus shuttle bus of UNDIP-UNNES, Semarang Indonesia

The trajectory which is passed by the bus in the simulation is double lane change. Fig 8 shows the relation between Simulink and TruckSim. The simulation is co-simulation, which means the both of software running at the same time. Input parameter for TruckSim is the torque from both motors which are located on the rear left and rear right. The outputs from
TruckSim are actual yaw rate, steering angle, longitudinal velocity, left and right rear wheel speed, rolling resistance of the left and right rear wheel. Longitudinal velocity, steering angle, and actual yaw rate are set as the input of the yaw control.

TABLE II BUS SPECIFICATION

\begin{tabular}{ccc}
\hline Parameter & Value & Unit \\
\hline Total Mass & 8140 & $\mathrm{Kg}$ \\
Passengers & 30 & Person \\
Length & 7500 & $\mathrm{~mm}$ \\
Width & 2100 & $\mathrm{~mm}$ \\
Height & 3330 & $\mathrm{~mm}$ \\
Floor Height & 900 & $\mathrm{~mm}$ \\
Wheelbase (L) & 3650 & $\mathrm{~mm}$ \\
C.G. to the rear wheel & 1817 & $\mathrm{~mm}$ \\
Maximum Speed & 70 & $\mathrm{~km} / \mathrm{h}$ \\
\hline Source: Measured, observed and estimated data from UNDIP-UNNES Bus
\end{tabular}

Simulation was run to achieve a double lane change maneuver of the bus. The time trace of actual and desired longitudinal velocities is shown in Fig 9 while the time trace of the steering angle and the wheel angle is shown in Fig 10 and Fig 11 respectively. The dynamic of the bus during this maneuver results to the yaw rate shown in Fig 12 in which the "desired" means the simulation result when both motors are always at the same rotational speed. The "actual" means the simulation result with the lateral controller with fuzzy logic is applied. The simulation result in Fig 12 demonstrates that they yaw rate has been reduced significantly with the lateral controller during the double lane change, while the actual tracking path can reasonably follow the desired one as shown in Fig 13.

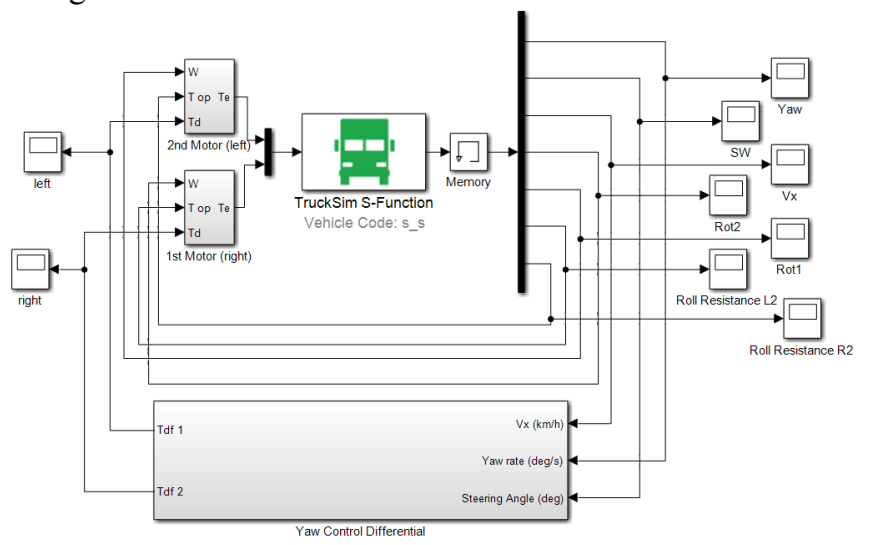

Fig 8 TruckSim and Simulink Co-simulation model 


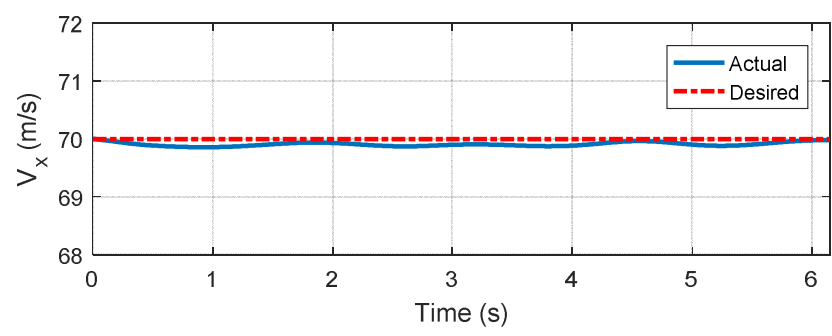

Fig 9 Longitudinal velocity

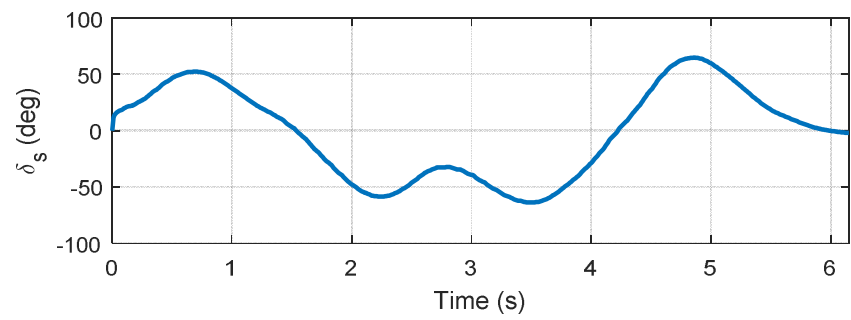

Fig 10 Steering angle

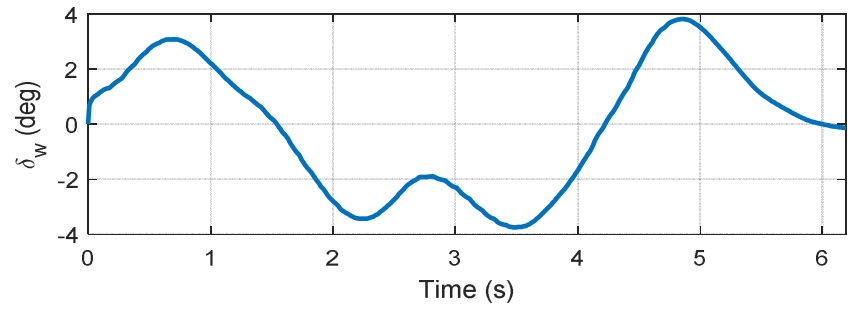

Fig 11 Wheel Angle

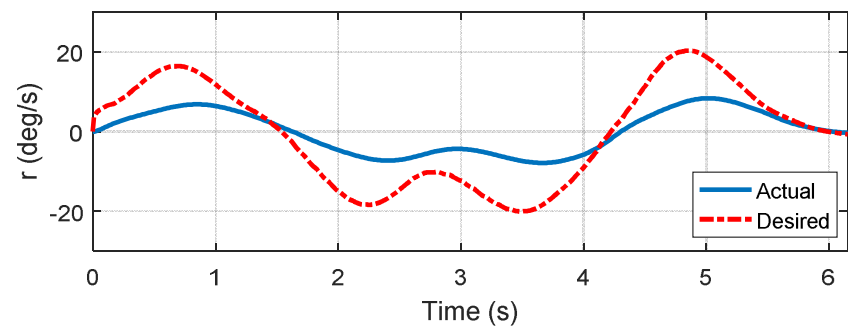

Fig 12 Yaw rate

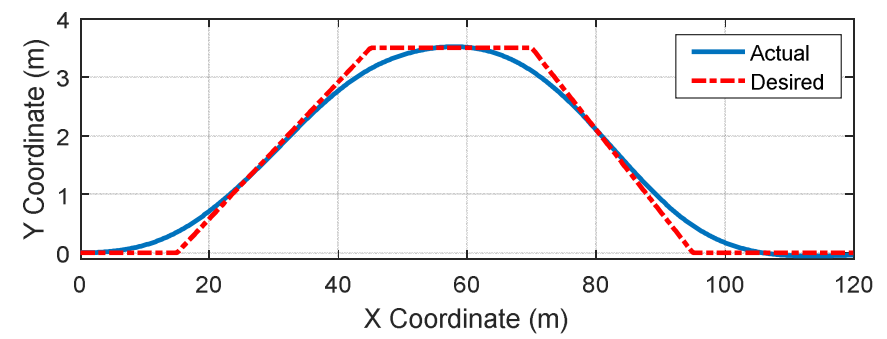

Fig 13 Tracking path

\section{CONCLUSION}

In this paper, the principle of the electronic differential to regulate dual motors was presented by considering the yaw rate of an electric urban bus. Dynamics model of the bus to generate the desired yaw rate was provided by TruckSim, and the control system was modeled in Matlab/Simulink. It was found that the bus had the characteristic speed of $150.36 \mathrm{~km} / \mathrm{h}$, with the wheel angle conditions of $10 \mathrm{deg}$ and the slip angle of $5 \mathrm{deg}$. The maximum difference between the desired yaw rate and the actual yaw rate was $12.635 \mathrm{deg} / \mathrm{s}$. During the bus maneuvers, it can be observed that the bus maintaines the speeds at $70 \mathrm{~km} / \mathrm{h}$ by using two independent motors and has reasonably path tracking response. The lateral controlled used in the simulation improves the dynamic stability of the bus.

\section{ACKNOWLEDGMENT}

This paper was supported by USAID through Sustainable Higher Education Research Alliances (SHERA) Program Centre for Collaborative (CCR) National Center for Sustainable Transportation Technology (NCSTT).

\section{REFERENCES}

[1] M. Mahmoud, R. Garnett, M. Ferguson, and P. Kanaroglou, "Electric buses: A review of alternative powertrains," Renew. Sustain. Energy Rev., vol. 62, pp. 673-684, Sep. 2016.

[2] Y. E. Zhao, J. W. Zhang, and X. Q. Guan, "Modeling and simulation of electronic differential system for an electric vehicle with twomotor-wheel drive," in 2009 IEEE Intelligent Vehicles Symposium, 2009, pp. 1209-1214.

[3] J. Y. Wong, Theory of ground vehicle 3rd Edition. New York: John Wiley and Sons, 2001.

[4] R. A. Rashid, J. D. Setiawan, M. Maharun, and M. B. Baharom, "Investigation Into the Effects of Maximum Deflection in Low Stiffness Resilient Shaft of Semi Active Steering Systems," ARPN J. Eng. Appl. Sci., vol. 11, no. 22, 2016.

[5] R. Rajamani, Vehicle Dynamics and Control. Boston, MA: Springer US, 2012.

[6] Y. Hua, H. Jiang, and G. Geng, "Electronic differential control of 2WD electric vehicle considering steering stability," in AIP Conference Proceedings, 2017, vol. 1820, no. 1, p. 070005.

[7] F. Dernoncourt, Introduction to fuzzy logic, vol. 21. 2013.

[8] F. Tahami, S. Farhangi, and R. Kazemi, "A Fuzzy Logic Direct YawMoment Control System for All-Wheel-Drive Electric Vehicles," Veh. Syst. Dyn., vol. 41, no. 3, pp. 203-221, Jan. 2004. 